\title{
Precision Calibration System for Partial Discharge Calibrators
}

\author{
J. Havunen \& J. Hällström \\ VTT Technical Research Centre of Finland Ltd, National Metrology Institute VTT MIKES, Finland \\ jussi.havunen@vtt.fi
}

\begin{abstract}
Partial discharge calibrators need to be periodically calibrated traceable to national standards. VTT MIKES, the National Metrology Institute (NMI) of Finland, has official expertise to calibrate the apparent charge of PD calibrators from $1 \mathrm{pC}$ to $100 \mathrm{nC}$ according to IEC 60270. Recently, VTT MIKES has developed techniques to measure charges as low as $0.01 \mathrm{pC}$ with $3 \%$ uncertainty $(k=2)$. VTT MIKES has participated to an intercomparison of four NMI's in 2018 and the results indicate that VTT MIKES can both expand their official measurement capabilities and lower their uncertainties. This paper presents VTT MIKES's precision calibration system for PD calibrators. Additionally, an overview of commercial PD calibrators' performance is provided and compared to the requirements of IEC 60270. Data is based on the calibrations performed during the last 10 years.
\end{abstract}

\section{Introduction}

Partial discharges (PD) are unwanted in high-voltage insulation systems because they may lead to flashover and unrecoverable damage of solid insulation. PD can be measured with suitable measuring systems. Measuring systems are calibrated separately for each measurement setup. Calibration is performed using a PD calibrator, which injects current pulses across the terminals of the test object. PD calibrators are widely available commercial devices that periodically need to be calibrated traceable to national standards. Commercial PD calibrators can have their lowest range as low as $0.1 \mathrm{pC}$, even though calibration services have not been available until recently.

IEC 60270:2000 [1] defines technical requirements for PD calibrators. The calibrators generate known charges by applying a step voltage to an injection capacitor, which is connected to the test object. Requirements for step voltage parameters, like rise time $t_{\mathrm{r}}$, time to steady state $t_{\mathrm{s}}$, and step voltage duration $t_{\mathrm{d}}$, are defined in the standard. In addition to the technical requirements, the standard also defines that performance tests should be performed periodically to PD calibrators. These tests should include the determination of the calibrator charge, rise time of the step voltage, and the pulse repetition rate. Three accepted calibration methods for the calibrator charge are presented in Annex A of the standard:

1. reference method;

2. numerical integration method; and

3. step voltage response method.

\section{Calibration system of VTT MIKES}

VTT MIKES is the National Metrology Institute (NMI) of Finland and one of its key tasks is to provide traceable calibration services to both domestic and international customers. At the moment VTT MIKES has official competence to calibrate the apparent charge of PD calibrators from $1 \mathrm{pC}$ to $100 \mathrm{nC}$ according to IEC 60270 using numerical and analog integration. The official calibration and measurement capabilities (CMCs) of VTT MIKES managed by the International Bureau of Weights and Measures (BIPM) are presented in Table 1 [2].

Table 1. CMCs of VTT MIKES for apparent charge.

\begin{tabular}{|c|c|}
\hline $\begin{array}{c}\text { Measurand level } \\
{[\mathbf{p C}]}\end{array}$ & Expanded uncertainty $(\boldsymbol{k}=\mathbf{2})$ \\
\hline $1-10$ & $0.2 \mathrm{pC}$ \\
\hline $10-100000$ & $20 \mathrm{mC} / \mathrm{C}$ \\
\hline
\end{tabular}

Currently the relative uncertainty is higher with the lower charges because uncertainties related to the low signal-to-noise ratio of the numerical integration method. The charge-sensitive preamplifier (CSP) based analog integration method for low charges is not yet described in our CMCs. The high signal-tonoise ratio of the CSP makes it possible to also calibrate the step voltage parameters with low uncertainty. Traceability of the two methods used are presented in Figure 1. All PD calibrations are traceable to SI via Finnish national standards of capacitance, DC voltage and resistance.

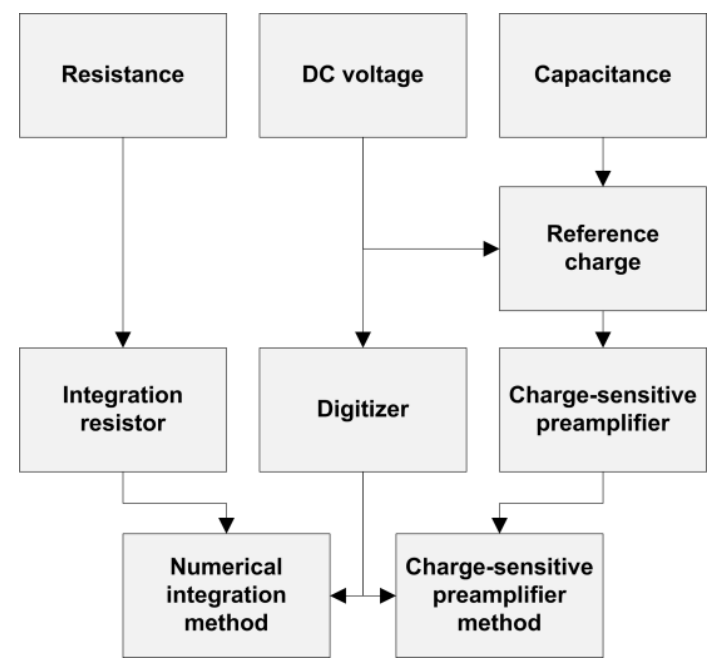

Figure 1. Traceability of the two calibration systems of VTT MIKES. 


\subsection{Generation of reference charge}

Reference calibrator is a device that is used to generate reference charge to determine the performance of different charge measuring systems. Basic principle of a reference calibrator is to apply a step voltage of amplitude $U_{0}$ in series with an injection capacitor $C_{0}$, which generates the reference charge $q_{0}$ so that:

$$
q_{0}=U_{0} C_{0}
$$

VTT MIKES approach for generation of the voltage step is to apply a DC voltage to the input terminal of the injection capacitor and then switch it to ground using a mercury-wetted relay. This method provides very fast rise and settling time of the step. The reference charge is applied from the output terminal of the injection capacitor to the measuring system. Polarity of the generated charge is inverse of the applied DC voltage.

Commercial DC voltage source is used together with an additional low-pass filter to stabilize the used voltage. Commercial reference voltmeter is used to measure the DC voltage with uncertainty less than $0.01 \%(k=2)$. Injection capacitors are shielded three-terminal capacitors, which are calibrated using a high-accuracy capacitance bridge. Uncertainty of the used capacitors are less than $0.2 \%(k=2)$. By combining different capacitor and voltage values a wide range of charges can be generated. Total expanded uncertainty of the reference calibrator of VTT MIKES is less than $0.2 \%(k=2)$. Similar uncertainties has also been reported by others [3]. This reference calibrator is used to calibrate the charge measuring systems of VTT MIKES.

\subsection{Measurements: numerical integration method}

Traditional method to measure the injected charge is to numerically integrate the current flowing through a resistor connected between the calibrator output and ground. This is approach is called the numerical integration method [1], which VTT MIKES is using for charges higher than $20 \mathrm{pC}$. This method is practical when the charges are not too low $(>10 \mathrm{pC})$ because the signal-to-noise ratio of the measurement is directly proportional to the applied charge. Millivolt-level signals are very difficult to measure accurately using a digitizer. This method does not allow to define the step voltage parameters defined in IEC 60270.

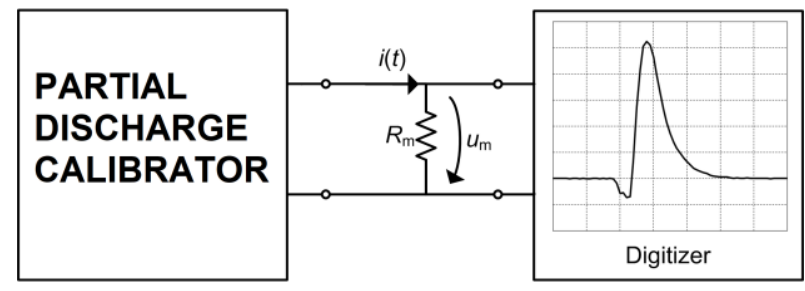

Figure 2. Main principle of the numerical integration method.
Charge $q_{0}$ from the PD calibrator is injected to a resistor connected in parallel to the digitizer high impedance input as seen in Figure 2. Current $i$ flowing through the resistor can be derived from the voltage $u_{\mathrm{m}}$ and resistor value $R_{\mathrm{m}}$. Charge $q_{0}$ can be numerically integrated from the current:

$$
q_{0}=\int i(t) d t=\frac{1}{R_{m}} \int u_{m}(t) d t .
$$

Integration resistor should be low-inductive with value from $50 \Omega$ to $200 \Omega$ [1]. VTT MIKES has several different self-built electrically shielded integration resistors in the specified range. Higher resistance values are typically used with the low charge levels in order to improve the signal level of the voltage impulse. However, due to the recent introduction of CSPs, the integration method is only used with the $50 \Omega$ resistor, which allows a decent signal level from $20 \mathrm{pC}$ upwards and keeps the voltage low enough for the used digitizer at $100 \mathrm{nC}$.

Used digitizer is an 8-bit with maximum sample rate of $4 \mathrm{GS} / \mathrm{s}$ and $1 \mathrm{GHz}$ bandwidth, which fulfils the minimum bandwidth requirement of $50 \mathrm{MHz}$ defined in IEC 60270 [1]. Digitizer is often used with $1 \mathrm{GS} / \mathrm{s}$ which often is enough to capture the impulse voltage to be integrated. Digitizer is calibrated in dynamic conditions by applying a known voltage step to its input [4]. Separate gain correction factors obtained from the calibration are used for every digitizer range.

Software is used to correct the gain of the digitizer range and to calculate the integral from the measured impulse. Defining and removing the offset level from the measured impulse is important in order to avoid integration errors [5]. Due to the lack of unambiguous instructions on defining the offset and the end of the integration period, different approaches might be applied by different calibration laboratories.

Estimated uncertainty $(k=2)$ for the system using numerical integration is approximately $2 \%$ in the range from $20 \mathrm{pC}$ to $100 \mathrm{nC}$. The main uncertainty components are the dynamic behaviour of the used digitizer, the uncertainties related to numerical integration of noisy sampled data and high standard deviation of the results.

\subsection{Measurements: analog integration method}

Integration of the injected current impulse can be also performed with hardware using analog integration $[4,6,7]$. This approach is not separately described in IEC 60270. However, it can be used according to the reference method [1], when the measurement system is calibrated against a reference calibrator. VTT MIKES's approach is to use CSPs to perform the analog integration and to amplify the signal [4].

Charge $q_{0}$ from the PD calibrator is injected to the input of the CSP. CSP is an integrating device which converts charge $q_{0}$ into voltage $U_{\mathrm{s}}$, which is measured from the output of the CSP using a digitizer. Because of the high gain of the CSP, the voltage can be measured accurately with a digitizer. Discharge of the feedback capacitor through the feedback resistor of the amplifier causes a drooping voltage output which is corrected with software [4]. Gain of the CSP is calibrated using a reference calibrator. The principle of the CSP is presented in Figure 3. 


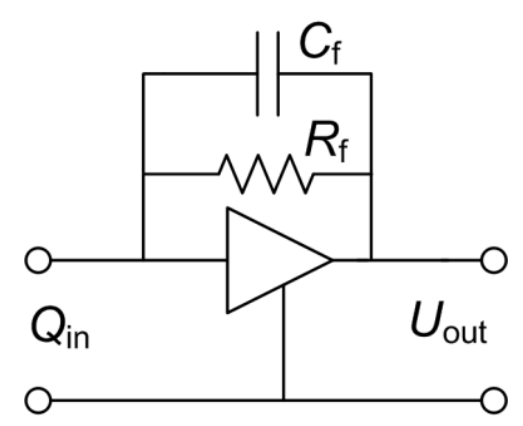

Figure 3. Main principle of the measuring system using CSP.

The CSP approach works well for low PD levels up to c. $100 \mathrm{pC}$. However, when trying to extend the method to higher charge levels the feedback capacitor value has to be increased. Higher capacitance values together with the need for fast $(<20 \mathrm{~ns})$ rise time set requirements to the amplifier design that cannot easily be met.

The digitizer is the same used in with the numerical integration method. Measured voltage impulse duration is microseconds while the rise times are nanoseconds. Due to these limitations the digitizer is commonly used with $500 \mathrm{MS} / \mathrm{s}$. Separate gain correction factors obtained from the calibration mentioned earlier are used for every digitizer range.

Same software is used with both numerical and analog integration. Numerical integration is not performed with CSP in use. Software is used to correct the gain of the digitizer range and the drooping response of the CSP. Voltage amplitude $U_{\mathrm{s}}$ of the measured step is calculated as the average value between 1 to $4 \mu \mathrm{s}$ after the step front [4]. $U_{\mathrm{s}}$ is divided by the gain of the CSP resulting the apparent charge $q_{0}$. Software can also be used to analyse the step voltage parameters defined in IEC 60270 [1].

Recently, VTT MIKES has demonstrated to measure $0.01 \mathrm{pC}$ with $3 \%$ uncertainty $(k=2)$ using CSP. Estimated uncertainty $(k=2)$ for the CSP method is less than $1 \%$ in the range of 0.1 to $20 \mathrm{pC}$. The main uncertainty components are the dynamic behaviour of the used digitizer and the uncertainty of the CSP calibration. Performance achieved using CSPs against current CMC entries are presented in Figure 4. VTT MIKES is currently applying a new CSP to expand their upper limit of the method from $20 \mathrm{pC}$ up to $200 \mathrm{pC}$.

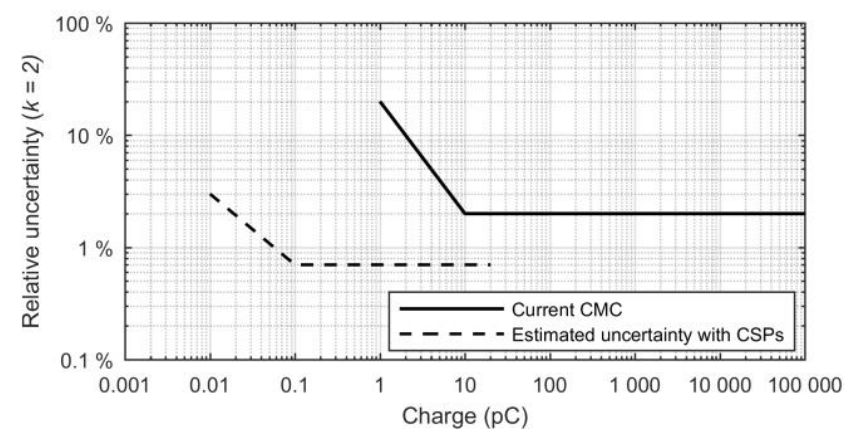

Figure 4. Calibration ranges and uncertainties of current CMC entry of VTT MIKES against the CSP method. [2]

\subsection{Current performance}

Four National Metrology Institutes (NMIs), including VTT MIKES, performed a comparison of their PD calibrator calibration capabilities in autumn 2018 [8]. Aim of this comparison was to confirm the existing measurement capabilities, and provide support for the extension to the lower charge levels. Step voltage parameters were also reported. Comparison provided experience for a more formal comparison, which is under consideration by the European Association of National Metrology Institutes (EURAMET).

Comparison was performed on charge levels from $0.1 \mathrm{pC}$ to $50 \mathrm{nC}$ produced by the transfer reference calibrator. Each lab provided their calibration results of the transfer reference calibrator together with their estimated uncertainties. VTT MIKES calibrated the transfer reference before (VTT1) and after (VTT2) the comparison. VTT1 results were selected as reference for presentation but they should not be taken as the comparison reference value. Results indicated that the difference between VTT1 and VTT2 was very small which indicates that the transfer reference has been very stable during the comparison. Other participants' results are presented anonymously in this paper.

Example of the comparison results with low charge levels is presented in Figure 5. Error bars present the claimed calibration uncertainty. The lowest charge levels are currently outside the official competence range of the institutes. It can be seen that institutes using charge amplifiers have significantly lower uncertainties than the institutes using the numerical integration method. For example, the claimed uncertainties $(k=2)$ of VTT MIKES for charges in the range from $0.1 \mathrm{pC}$ to $20 \mathrm{pC}$ were less than $1 \%$. The results support the improved measurement capabilities and uncertainty claims of the participating institutes well. In addition to the low charge levels, results for the charges from $10 \mathrm{pC}$ to $50 \mathrm{nC}$ are well within $2 \%$, which is the lowest official uncertainty of the participating institutes according to their CMCs. Reported step voltage parameters were also rather well in line with the participating institutes.

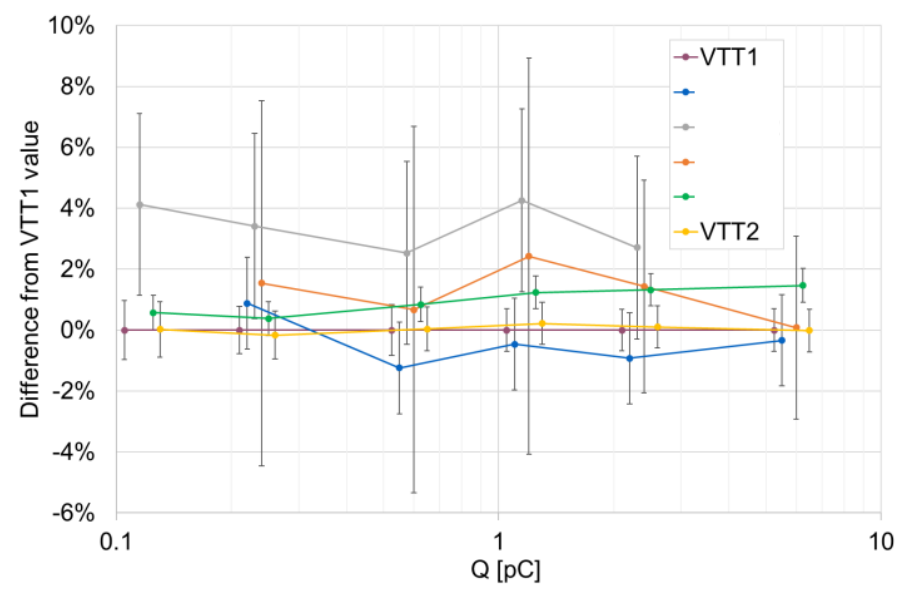

Figure 5. Example of the comparison results in range $0.1 \mathrm{pC}$ to $5 \mathrm{pC}$. [6]. Small horizontal offsets have been applied to results for clarity of presentation. 


\section{Overview of the performance of the commercial PD calibrators}

According to IEC 60270 the calibration of the calibrator charge should be performed with an uncertainty better than $5 \%$ or $1 \mathrm{pC}$, whichever is greater. Calibrators should be used with the calibrated value, and no special acceptance limit for deviation from the nominal is given in the standard [1]. However, if the nominal value of charge is used with the calibrator, then the uncertainty limit given in the standard can be considered as an acceptance limit. This kind of approach was used in this study to evaluate to the performance of commercial PD calibrators.

Data from commercial PD calibrators calibrated at VTT MIKES were used to analyze their performance. Data is based on the calibrations from the last 10 years, which are mainly performed using the numerical integration method with an expanded uncertainty $(k=2)$ of $2 \%$ or $0.2 \mathrm{pC}$ whichever is greater. All presented charge levels are absolute values so that they include both positive and negative polarity. Calibration results for all analyzed test points are presented in Figure 6. Total number of calibration values is 598 .

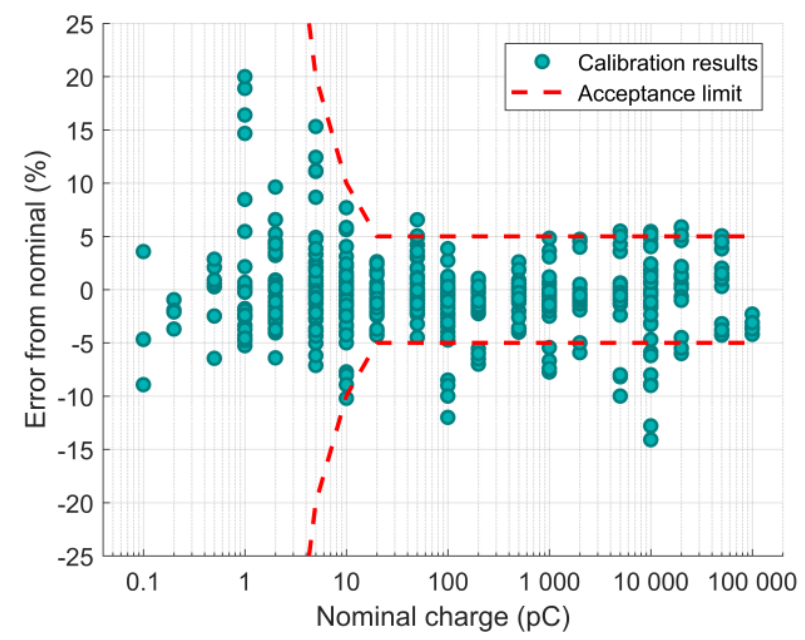

Figure 6. Graphic presentation of the calibration results of commercial PD calibrators calibrated at VTT MIKES. Used acceptance limit was $5 \%$ or $1 \mathrm{pC}$, whichever is greater.

In approximately $1 \%$ of the cases the calibrator (or its setting) was found broken, and calibration was not possible. In approximately $7 \%$ of the cases the result was outside the used acceptance limit, when calibration uncertainty is not taken account. If the calibration uncertainty is taken account, the analysis will become more difficult because some results outside the acceptance limit can be inside the limit and vice versa. For this purpose, it was found practical to ignore the measurement uncertainties for this analysis.

In most cases where the results were outside the acceptance limit, the generated charge was too small, which can be seen in Figure 6. Average error of all results was $-0.7 \%$ which shows that the errors of an average PD calibrator are slightly biased to the negative side. However, the average error is within the calibration uncertainties.
Calibration data also shows that most of the test points have been between $1 \mathrm{pC}$ and $10 \mathrm{nC}$ while the lowest and highest charges have been special cases. However, we have seen increasing interest in calibration of the extreme values.

\section{Conclusions}

Calibration system of VTT MIKES for PD calibrators was described. Recently introduced approach using charge-sensitive preamplifiers both expands the measuring range and reduces uncertainties for low charge levels. With the new approach the calibrations can be performed down to $0.01 \mathrm{pC}$, and the uncertainty can be lowered down to $1 \%$ for low charge levels between 0.1 and $20 \mathrm{pC}$. Comparison to other NMIs supports the claimed performance.

Analysis of the calibrated commercial PD calibrators highlights the importance of periodical calibration to reveal devices that have large errors compared to their nominal values, or are broken.

\section{References}

[1] IEC 60270:2010+AMD1:2015, "High-voltage test techniques - Partial discharge measurements", 2015.

[2] Bureau International des Poids et Mesures. Calibration and Measurement Capabilities, Electricity and Magnetism. Accessed: March. 22, 2019. [Online]. Available: http://kcdb.bipm.org/appendixC/search.asp?reset= $1 \&$ met=EM

[3] O. Gunnarson, A. Bergman and K.-E. Rydler. "A method for calibration of partial discharge calibrators", IEEE Transactions on Instrumentation and Measurement, Vol. 48, No. 2, April 1999, pp. 453-456.

[4] J. Havunen and J. Hällström, “Application of charge-sensitive preamplifier for the calibration of partial discharge calibrators below $1 \mathrm{pC}$ ", 12.2.2019, IEEE Transactions on Instrumentation and Measurement, 7 p. doi: 10.1109/TIM.2019.2895437

[5] R. Gobbo, G. Pesavento, A. Sardi, G. Varetto, C. Cherbaucich and G. Rizzi. "Influence quantities in the calibration of PD calibrators contribution to the uncertainty estimate". Eleventh International Symposium on High Voltage Engineering, 23.-27.8.1999, London, United Kingdom, Conference Proceedings, pp. 5.272.P5-5.275.P5.

[6] K. Drazba, R. Grychowski, S. Drazba, and R. Malewski, "Performance tests of PD measuring instruments and calibrators," in Proc. Conf. ERA98 Meas. Calibration High Voltage Test., London, U.K., 1998, pp. 3.3.1-3.3.8.

[7] W. Hauschild and E. Lemke, High-voltage Test and Measuring Techniques. Berlin, Germany: Springer, 2014, pp. 186-189.

[8] J. Hällström, J. Havunen, A.E. Bergman, A-P. Elg, A. Merev, S. Dedeoğlu, İ. Karaman, P. Simón, J. Rovira, T. Garcia and F. Garnacho, "Comparison of PD calibration capabilities in four National Metrology Institutes down to 0.1 pC", $21^{\text {st }}$ International Symposium on High Voltage Engireering, ISH 2019, Budapest, Hungary, August 26-30, 2019. Accepted for presentation. 